\title{
Use of Phthalimidoacetyl Isothiocyanate as a Scaffold in the Synthesis of Target Heterocyclic Systems, and Their Antimicrobial Assessment
}

\author{
Magdy Mohamed Hemdan* and Amira Abd-Elhaleem El-Sayed \\ Department of Chemistry, Faculty of Science, Ain Shams University; Abbasia, Cairo 11566, Egypt. \\ Received January 31, 2016; accepted February 17, 2016
}

\begin{abstract}
Phthalimidoacetyl isothiocyanate underwent addition-cyclization reactions with some nitrogen and carbon nucleophilic reagents. Simultaneous or subsequent cyclization of the obtained adducts gave target heterocyclic systems such as 1,2,4-triazoles, 1,3-diazines, 1,3-oxazines and thiourea attached to a phthalimido moiety. The antimicrobial activity of the synthesized compounds was tested.
\end{abstract}

Key words phthalimidoacetyl isothiocyanate; 1,2,4-triazol; 1,3-diazine; 1,3-oxazine; thiourea; antimicrobial

Compounds containing phthalimido moiety have been described as a scaffold to design new prototypes of drugs with different biological activities. Some of them are used for treatment of different diseases such as infectious diseases, ${ }^{1)}$ tuberculosis, ${ }^{2)}$ AIDS, ${ }^{3)}$ tumors, ${ }^{4)}$ multiple myeloma, ${ }^{5)}$ inflammatory diseases, ${ }^{6)}$ asthma, ${ }^{7}$ diabetes, ${ }^{8)}$ hyperlipidemia, ${ }^{9}$ convulsion, ${ }^{10}$ depression. ${ }^{11)}$ Recently, they are evaluated as anti-Alzheimer's agents. ${ }^{12)}$ Moreover, the biological activities of 1,2,4-triazoles, ${ }^{13)} 1,3$-diazines $,{ }^{14)} 1,3$-oxazines, ${ }^{15)}$ thioureas ${ }^{16}$ are well known.

In the past few years the authors have been involved in the uses of aroyl isothiocyanates ${ }^{17-20)}$ and acyl isothiocyanates ${ }^{21-24)}$ in different synthetic strategies of target heterocycles. Studies on the chemistry of aroyl and/or acyl isothiocyanates have established the value of these compounds as starting materials in the synthesis of a wide variety of heterocyclic systems and thiourea derivatives. ${ }^{17-27)}$

Based on the previous work, we have been synthesized variety of heterocyclic systems bearing phthalimido moiety by using 2-phthalimidobenzoyl isothiocyanate ${ }^{19)}$ and phthalimidoacyl isothiocyanate. ${ }^{22)}$ In the current study, a further stage reactions of phthalimidoacetyl isothiocyanate was performed.

\section{Results and Discussion}

Treatment of a solution of phthalimidoacetyl isothiocyanate 1 in a dry acetonitrile with 2-cyanoacetohydrazide at room temperature gave thiosemicarbazide derivative $\mathbf{2}$ in a good yield. On the other hand, the reaction of a mixture of isothiocyanate $\mathbf{1}$ and 2-cyanoacetohydrazide in acetonitrile under refluxing conditions produced 1,2,4-triazole derivative 3 in one pot-reaction. However, boiling a solution of the adduct 2 in acetonitrile also gave the 1,2,4-triazole derivative $\mathbf{3}$. Refluxing of compound $\mathbf{3}$ in ethanolic-hydrochloric acid mixture afforded ester derivative 4 . On the other hand, when a solution of $\mathbf{3}$ in ethanol was boiled with a catalytic amount of sodium hydroxide, followed by acidification with dilute $\mathrm{HCl}$ furnished a carboxylic acid derivative $\mathbf{6}$ as illustrated in Chart 1.

The structures of the synthesized compounds 2-4 and 6 were elucidated from their microanalytical and spectral data. Thus, their IR spectra displayed bands corresponding to $\mathrm{NH}$, $\mathrm{CO}$ and $\mathrm{C}=\mathrm{S}$, as well as stretching band of $\mathrm{CN}$ group for compounds 2 and 3. The ${ }^{1} \mathrm{H}-\mathrm{NMR}$ spectra of the synthesized compounds are in accord with their proposed structures as they showed signals for aromatic protons and aliphatic protons in addition to $\mathrm{NH}$ protons in the downfield region exchangeable with $\mathrm{D}_{2} \mathrm{O}$. The appearance of an exchangeable broad singlet signal for compound $\mathbf{4}$, at $\delta$ : $7.16 \mathrm{ppm}$ corresponds to $\mathrm{SH}$ proton suggests its existence entirely as aromatic triazole structure. The relatively high $\delta$ value of $\mathrm{SH}$ proton suggests chelation shown (Chart 1). The ${ }^{1} \mathrm{H}-\mathrm{NMR}$ spectrum of compound 6 showed the absence of a signal corresponding to $\mathrm{CH}_{2}$ protons in the up field region, instead it revealed two olefinic signals at $\delta$ : 5.88 and $5.94 \mathrm{ppm}$ integrated to one proton. These observations suggest that the existence of compound 6 as $E / Z$ mixture $\mathbf{6 a}$ and $\mathbf{b}$ in the ratio $3: 97$, respectively. Compound $\mathbf{6}$ gets its stabilization from the formation of conjugated $\alpha$-enone system. The higher ratio of Z-configurated isomer as compared with $E$-counterpart can be rationalized on the basis of stabilization through chelation shown in structure 6b. Moreover, the ${ }^{13} \mathrm{C}-\mathrm{NMR}$ spectra of compounds $\mathbf{4}$ and $\mathbf{6}$ support their proposed structures (see Experimental). Further highlights on the assigned structures were gained from their mass spectral data that showed the correct molecular ion peaks and the mass of fragment peaks in agreement with their proposed structures. The synthetic strategy towards the synthesis of 1,2,4-triazolethione derivatives 3 involves the addition of 2-cyanoacetohydrazide to the electrophilic carbon of isothiocyanate $\mathbf{1}$ to give thiosemicarbazide derivative $\mathbf{2}$. The latter undergoes cyclization to give $\mathbf{3}$ via intramolecular nuleophilic addition of $\mathrm{NH}$ to $\mathrm{C}=\mathrm{O}$ group followed by elimination of water molecule [exo-trig cyclization] (Chart 1). Compounds 4 and 6 were visualized as a hydrolyzed product of compound 3 , followed by esterification to give compound $\mathbf{4}$ or rearrangement of the intermediate 5 to more stable $\alpha$-enone structure $\mathbf{6}$.

The reaction of isothiocyanate $\mathbf{1}$ with thiourea in a dry acetonitrile produced 1-(2-(1,3-dioxoisoindolin-2-yl)acetyl)thiourea 7 in a good yield. On the other hand refluxing of 1 with thiosemicarbazide in acetonitrile was accompanied by release of $\mathrm{H}_{2} \mathrm{~S}$ gas and leave a product formulated as 1,2,4-triazole derivative 9 as shown in Chart 2. The interaction of equimolar quantities of $\mathbf{1}$ and ethyl carbazate in acetonitrile gave a good yield of the linear 1:1 adduct 10. Compound $\mathbf{1 0}$ resists cyclization even under refluxing condition for a long time. However refluxing a solution of compound $\mathbf{1 0}$ in $\mathrm{HCl}-$ ethanol mixture effected ring closure with elimination of ethanol molecule to give 1,2,4-triazole derivative $\mathbf{1 2}$. 

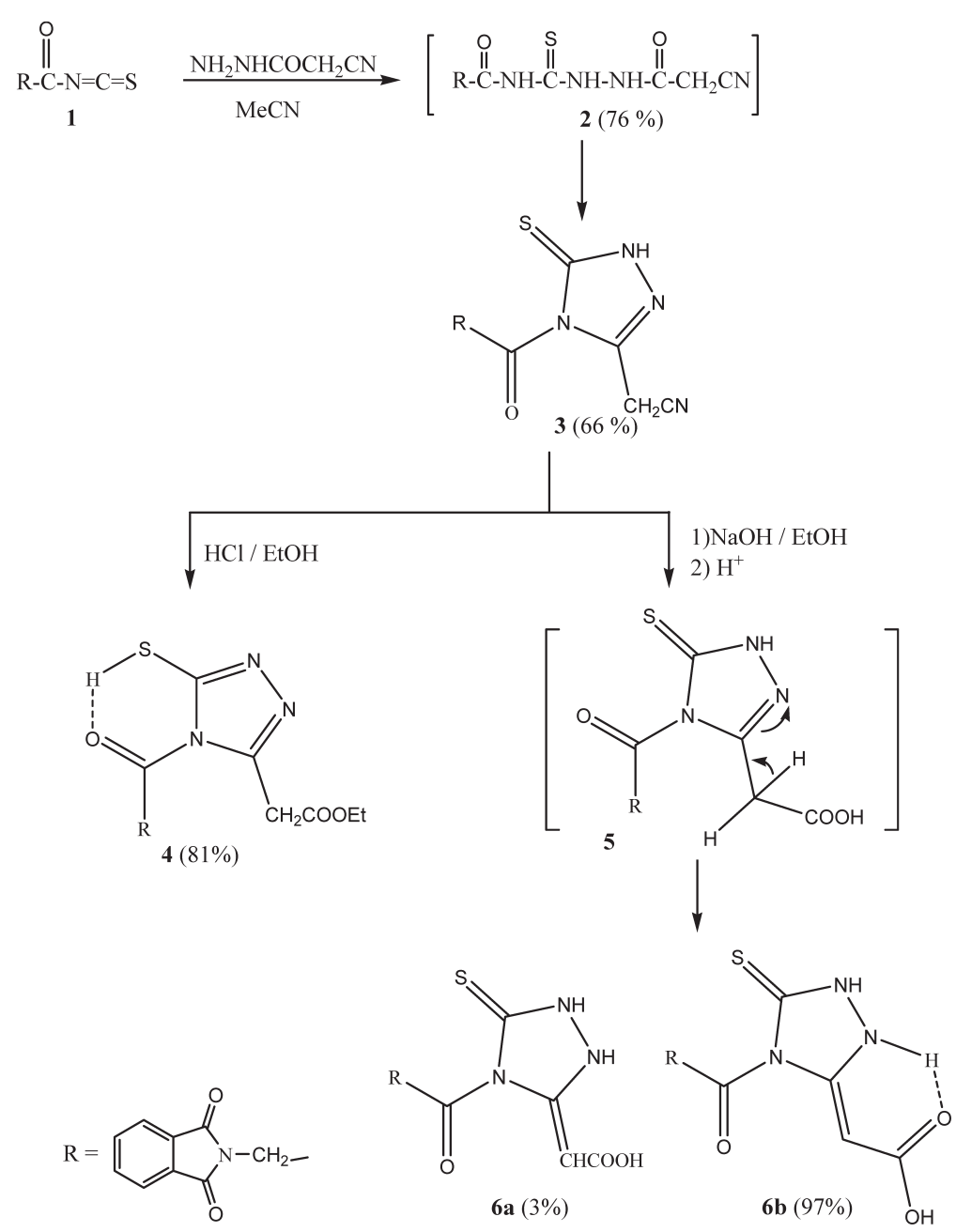

Chart 1. Reaction of Phthalimidoacetyl Isothiocyanate $\mathbf{1}$ with 2-Cyanoacetohydrazide and Cyclisation of the Products with Ethanolic Solution of $\mathrm{HCl}$ and $\mathrm{NaOH}$

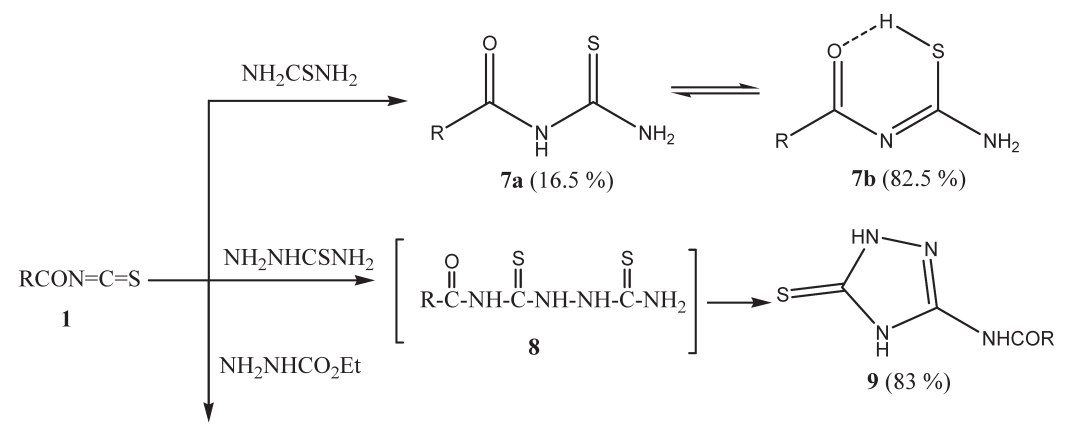

$\stackrel{\mathrm{O}}{\mathrm{R}} \stackrel{\mathrm{C}}{\mathrm{S}-\mathrm{NH}-\mathrm{C}-\mathrm{NH}-\mathrm{NH}-\mathrm{C}-\mathrm{OEt}}$

$$
10(78 \%)
$$

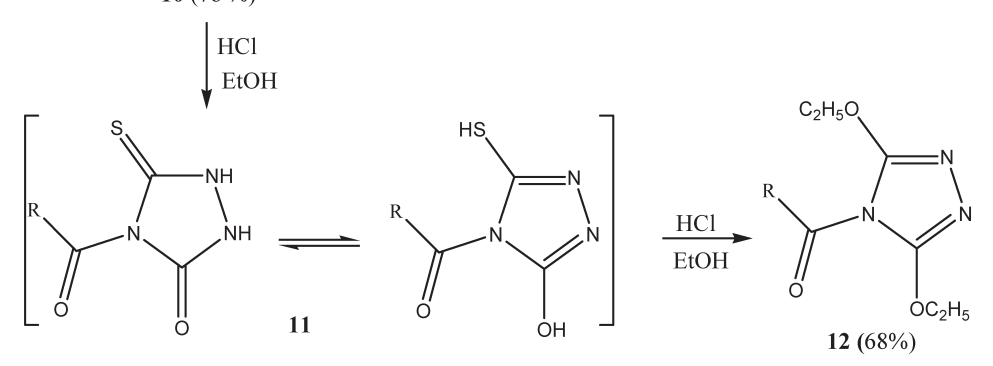

Chart 2. Reaction of $\mathbf{1}$ with Thiourea, Thiosemicarbazide and Ethyl Carbazate 


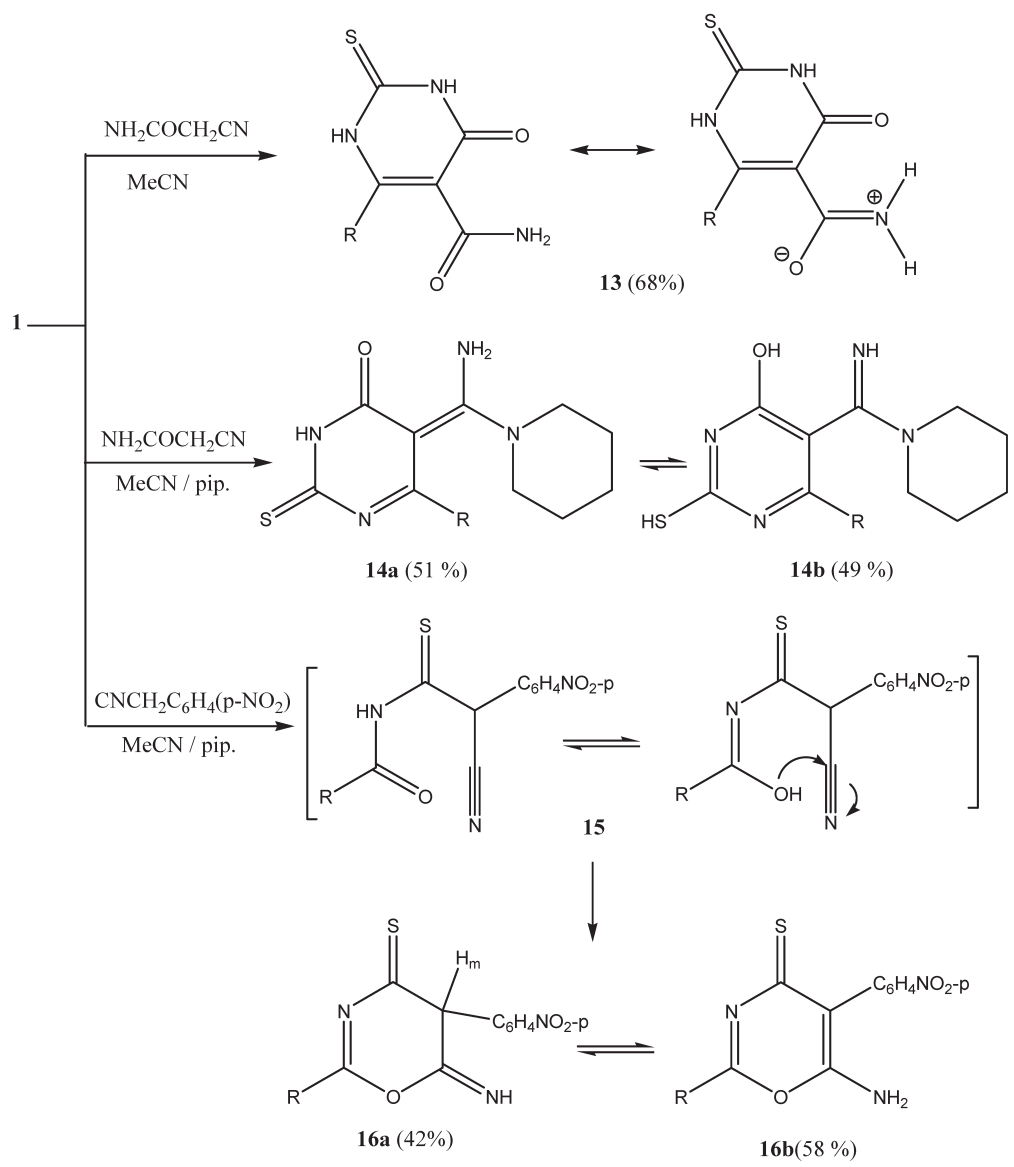

Chart 3. Reaction of $\mathbf{1}$ with 2-Cyanoacetamide and $p$-Nitrobenzylcyanide

The structures of the synthesized compounds $7,9,10$ and 12 were elucidated from their microanalytical and spectral data. Thus, their IR spectra showed doublet bands in the region $\left(1765-1710 \mathrm{~cm}^{-1}\right)$ characteristic for coupling carbonyl of imides and in addition to the bending vibration of $\mathrm{C}-\mathrm{H}$ bonds of the methylene group in the region $1473-1417 \mathrm{~cm}^{-1}$. Their ${ }^{1} \mathrm{H}-\mathrm{NMR}$ spectra displayed signals for aliphatic and aromatic protons beside $\mathrm{NH}$ protons in the down field region that were exchangeable with $\mathrm{D}_{2} \mathrm{O}$. The ${ }^{1} \mathrm{H}-\mathrm{NMR}$ spectrum of compound 7 showed its existence as mixture of thione-thiol tautomers $7 \mathbf{a}$ and $\mathbf{b}$ in the ratio of $1: 5$. The higher ratio of the isomer $\mathbf{7 b}$ may be attributed to its chleated form in DMSO$d_{6}$ solution. The ${ }^{13} \mathrm{C}$-NMR spectra of compounds 7, 9 and $\mathbf{1 2}$ are displayed in Experimental and they are in agreement with their proposed structures. The reaction of isothiocyanate $\mathbf{1}$ with thiourea takes place via nucleophilic addition of thiourea on isothiocyanate $\mathbf{1}$ to give nonisolable intermediate [ $\mathrm{R}-\mathrm{CO}-$ $\mathrm{NH}-\mathrm{CS}-\mathrm{NH}-\mathrm{CS}-\mathrm{NH}_{2}$ ] followed by extrusion of isothiocyanic acid molecule to give 7 . Compound 9 is thought to be formed by elimination of $\mathrm{H}_{2} \mathrm{~S}$ molecule from the non-isolable intermediate 8 via exo-trig cyclization of $\mathrm{NH}$ group on $\mathrm{C}=\mathrm{S}$ group followed by elimination of $\mathrm{H}_{2} \mathrm{~S}$. In case of formation of compound 12, $\mathrm{HCl}$ catalyzes exo-trig cyclization of $\mathrm{NH}$ group on the $\mathrm{C}=\mathrm{O}$ group of ester followed the removal of ethanol molecule from $\mathbf{1 0}$ to give the non-isolable intermediate $\mathbf{1 1}$ that converted to compound $\mathbf{1 2}$ under the reaction condition.

Isothiocyanate 1 underwent reaction with 2-cyanoacetamide in boiling acetonitrile to yield pyrimidine derivative 13. Refluxing of $\mathbf{1}$ with 2-cyanoacetamide in the presence of an equivalent amount of piperidine yielded pyrimidine derivative 14. The reaction of $\mathbf{1}$ with $p$-nitrobenzylcyanide afforded 1,3-oxazine derivatives $\mathbf{1 6}$. The ${ }^{1} \mathrm{H}$-NMR spectrum of 13 showed its amide $\mathrm{NH}_{2}$ protons as two singlet signals at $\delta$ : $7.81,7.63$, which suggests their magnetically nonequivalence property. The ${ }^{1} \mathrm{H}-\mathrm{NMR}$ spectrum of $\mathbf{1 4}$ showed its existence in dimethyl sulfoxide (DMSO) solution as an equilibrium mixture of 14a and $\mathbf{b}$ in approximately equal ratio $51: 49$. The ${ }^{1} \mathrm{H}-\mathrm{NMR}$ spectrum of compound $\mathbf{1 6}$ showed its existence as an equilibrium mixture of 16a and $\mathbf{b}$ in the ratio $42: 58$ respectively. The formation of compounds $\mathbf{1 3}$ and $\mathbf{1 4}$ can be explained on the basis of cyclocondensation of $\mathbf{1}$ with 2-cyanoacetamide followed by partial hydrolysis of nitrile group to produce amide derivative $\mathbf{1 3}$ or nucleophilic addition of piperidine nitrogen atom to the electron deficient carbon atom of nitrile group to give compound $\mathbf{1 4}$. Compound $\mathbf{1 6}$ can be demonstrated on the basis of addition of the carbanion of $p$ nitrobenzylcyanide to isothiocyanate carbon atom of $\mathbf{1}$ to give the non-isolable intermediate $\mathbf{1 5}$, followed by exo-dig cyclization as shown in Chart 3.

Screening of the Antimicrobial Activity of the Chemically Synthesized Compounds The newly synthesized compounds were screened for their antibacterial and antifungal activities using the agar well diffusion technique. The microorganisms (reference and clinical isolates) used include Gramnegative Escherichia coli (ATCC-25922) and Salmonella typhi, Gram-positive Staphylococcus aureus (ATCC-25923), fungi Candida albicans (ATCC-10231) and Aspergillus flavus as shown in the results of Table 1. The study also included the 
activity of reference compounds sulphamethoxazole as antibacterial agent and fluconazole as antifungal agent.

The tested compounds 2-4, 6, 9, 10, 12-14 and 16 are exhibited a high activity against both Escherichia coli (ATCC-25922) and Salmonella typhi (as examples of Gramnegtive), Staphylococcus aureus (ATCC-25923) (as example of Gram-positive), Candida albicans (ATCC-10231) (pathogenic yeast) and Aspergillus flavus (pathogenic mold). The tested compounds showed zone of inhibition diameters ranged from 30 to $34 \mathrm{~mm}$ against E. coli, 28 to $34 \mathrm{~mm}$; against $S$. typhi and 32 to $36 \mathrm{~mm}$ against $S$. aureus, at $500 \mu \mathrm{g} / \mathrm{mL}$ of DMSO. In comparison with fluconazol, the tested compounds were showed zone of inhibition diameters ranged from 21 to $25 \mathrm{~mm}$ and 22 to $25 \mathrm{~mm}$ against $C$. albicans and A. flavus, respectively at $500 \mu \mathrm{g} / \mathrm{mL}$ of DMSO. The compound 2 revealed a highest activity against $S$. aureus $(36 \mathrm{~mm})$. Evident minimum inhibitory concentration (MIC) values on the entire set of the tested microbial organism were determined for the chemical agents $\mathbf{2}-\mathbf{4}, \mathbf{6}, \mathbf{9}, \mathbf{1 0}, \mathbf{1 2}-\mathbf{1 4}$ and $\mathbf{1 6}$ and the results are summarized in Table 2. The MIC values are ranged from 6.25 to $12.5 \mu \mathrm{g} / \mathrm{mL}$ in case of the chemically synthesized compounds against the used microbial. Compounds $\mathbf{2}$ and $\mathbf{6}$ are the most potent compounds.

\section{Experimental}

General Melting points were measured on an electrothermal melting point apparatus and were uncorrected. The elemental analyses were done on a Perkin-Elemer $2400 \mathrm{CHN}$ elemental analyzer. The IR spectra were recorded on Fourier transform (FT)-IR Maltson (infinity series) spectrophotometer as $\mathrm{KBr}$ discs. The ${ }^{1} \mathrm{H}-\mathrm{NMR}$ spectra were measured on Varian Gemini $300 \mathrm{MHz}$ spectrometer, with chemical shift $(\delta)$ expressed in ppm downfield from tetramethylsilane (TMS) as internal standard, in DMSO- $d_{6}$. Mass spectra were determined on Shimadzu GC-MSQP 1000 EX instrument operating at $70 \mathrm{eV}$. TLC was run using TLC aluminum sheets silica gel $\mathrm{F}_{254}$ (Merck). It was carried out the monitoring of the progress of all reactions and homogeneity of the synthesized compounds. Phthalimidoacetyl isothiocyanate was prepared according to literature $^{22)}$ and was used in situ.

Synthesis of 1-(2-Cyanoacetyl)-4-(2-(1,3-dioxoisoindolin-2-yl)acetyl)thiosemicarbazide (2) To a solution of phthalimidoacetyl isothiocyanate (1) $(3 \mathrm{mmol})$ in a dry acetonitrile $(30 \mathrm{~mL}), 2$-cyanoacetohydrazide $(3 \mathrm{mmol})$ was added. The reaction mixture was stirred at room temperature for 2h. A solid product was obtained during stirring that was filtered off and recrystallized from ethanol to give compound 2. Seventy-six percent yield; pale yellow crystals; mp 197-199 ${ }^{\circ}$; ${ }^{1} \mathrm{H}-\mathrm{NMR}$ (DMSO- $\left.d_{6}\right) \delta: 3.81\left(2 \mathrm{H}, \mathrm{s}, \mathrm{CH}_{2} \mathrm{CN}\right)$,

Table 1. Antimicrobial Activity of Chemically Synthesized Compounds

\begin{tabular}{|c|c|c|c|c|c|}
\hline No. & E. coli & S. typhi & S. aureus & C. albicans & A. flavus \\
\hline 2 & 34 & 31 & 36 & 23 & 25 \\
\hline 3 & 31 & 29 & 32 & 21 & 22 \\
\hline 4 & 33 & 34 & 33 & 20 & 24 \\
\hline 6 & 33 & 30 & 34 & 25 & 25 \\
\hline 9 & 32 & 30 & 33 & 23 & 23 \\
\hline 10 & 32 & 33 & 34 & 23 & 24 \\
\hline 12 & 30 & 28 & 34 & 22 & 24 \\
\hline 13 & 32 & 30 & 34 & 21 & 25 \\
\hline 14 & 33 & 28 & 34 & 22 & 24 \\
\hline 16 & 31 & 28 & 32 & 23 & 25 \\
\hline $\mathrm{S}$ & 38 & 35 & 35 & $\mathrm{~N}$ & $\mathrm{~N}$ \\
\hline $\mathrm{F}$ & $\mathrm{N}$ & $\mathrm{N}$ & $\mathrm{N}$ & 30 & 27 \\
\hline
\end{tabular}

$\mathrm{S}=$ Sulfamethoxazol $10 \mu \mathrm{g} / \mathrm{mL}$ (antibacterial agent); $\mathrm{F}=$ Fluconazol $10 \mu \mathrm{g} / \mathrm{mL}$ (antifungal agent). The concentration of all synthesized compounds were (500 $\mu \mathrm{g} / \mathrm{mL}$ in DMSO); $0.0=$ no inhibition. $\mathrm{N}=$ not tested.

Table 2. Minimum Inhibition Concentration (MIC) of the Chemically Synthesized Compounds

\begin{tabular}{|c|c|c|c|c|c|}
\hline \multirow{2}{*}{ No. } & \multicolumn{5}{|c|}{ MIC values $(\mu \mathrm{g} / \mathrm{mL})$} \\
\hline & E. coli & S. typhi & S. aureus & C. albicans & A. flavus \\
\hline 2 & 6.25 & 12.5 & 6.25 & 12.5 & 6.25 \\
\hline 3 & 12.5 & 12.5 & 12.5 & 6.25 & 12.5 \\
\hline 4 & 12.5 & 12.5 & 6.25 & 6.25 & 12.5 \\
\hline 6 & 6.25 & 12.5 & 6.25 & 6.25 & 12.5 \\
\hline 9 & 12.5 & 12.5 & 12.5 & 12.5 & 12.5 \\
\hline 10 & 12.5 & 25 & 12.5 & 12.5 & 12.5 \\
\hline 12 & 12.5 & 12.5 & 6.25 & 12.5 & 12.5 \\
\hline 13 & 12.5 & 25 & 12.5 & 12.5 & 12.5 \\
\hline 14 & 12.5 & 12.5 & 12.5 & 12.5 & 12.5 \\
\hline 16 & 12.5 & 12.5 & 12.5 & 12.5 & 12.5 \\
\hline $\mathrm{S}$ & 3.125 & 3.125 & 3.125 & $\mathrm{~N}$ & $\mathrm{~N}$ \\
\hline $\mathrm{F}$ & $\mathrm{N}$ & $\mathrm{N}$ & $\mathrm{N}$ & 3.125 & 3.125 \\
\hline
\end{tabular}


4.55 (2H, s, $\left.\mathrm{CH}_{2} \mathrm{CO}\right), 7.88-7.96$ (4H, m, ArH), $11.10(1 \mathrm{H}, \mathrm{brs}$, NHCS, exchangeable), 11.80 (1H, brs, NHCO, exchangeable), 12.02 (1H, brs, CONHCS, exchangeable); IR (KBr) v: 3335, 3237, $3155(\mathrm{NH}), 305 \overline{4}\left(\mathrm{C}-\mathrm{H}_{\text {arom }}\right), 2944,2908\left(\mathrm{C}-\mathrm{H}_{\text {aliph }}\right), 2265$ $(\mathrm{CN}), 1773,1720(\mathrm{C}=\mathrm{O}), 1196(\mathrm{C}=\mathrm{S}), 1467\left(\delta_{\mathrm{s}} \mathrm{CH}_{2}\right), 757 \delta_{4 \mathrm{H}}$; MS (70 eV) m/z (\%): $345\left(\mathrm{M}^{+}, 1\right), 327$ (3), 286 (8), (3), 260 (93), 242 (20), 188 (68), 160 (100), 133 (85), 104 (82), 77(67); Anal. Calcd for $\mathrm{C}_{14} \mathrm{H}_{11} \mathrm{~N}_{5} \mathrm{O}_{4} \mathrm{~S}$ (345.33): C, 48.69; H, 3.21; N, 20.28. Found C, 48.39; H, 2.98; N, 20.12\%.

Uses of Isoyhiocyanate $\mathbf{1}$ in Syntheses of Compounds 3, 7, 9, 10 and 13

General Procedure A mixture of phthalimidoacetyl isothiocyanate (1) $(3 \mathrm{mmol})$ and 2-cyanoacetohydrazide $(3 \mathrm{mmol})$ in a dry acetonitrile $(30 \mathrm{~mL})$ was refluxed for $3 \mathrm{~h}$. The reaction mixture was cooled to room temperature to give a solid product that was filtered off and recrystallized from ethanol to give compound $\mathbf{3}$. Also, compound $\mathbf{3}$ was obtained from refluxing of thiosemicarbazide derivative $2(0.5 \mathrm{~g})$ in acetonitrile $(20 \mathrm{~mL})$ for $2 \mathrm{~h}$. The same procedure was done with thiourea, thiosemicarbazide, ethyl carbazate, and/or 2-cyanoacetamide to give compounds $\mathbf{7 , 9 , 1 0}$ and $\mathbf{1 3}$, respectively. The progress of the reactions and homogeneity of the synthesized compounds were monitored by TLC. The solid obtained for each reaction was recrystallized from a suitable solvent to give the corresponding compound.

2-(4-(2-(1,3-Dioxoisoindolin-2-yl)acetyl)-5-thioxo-4,5dihydro-1 $H$-1,2,4-triazol-3-yl)acetonitrile (3)

Sixty-six percent yield; colorless crystals; mp $275-277^{\circ} \mathrm{C}$; ${ }^{1} \mathrm{H}-\mathrm{NMR}\left(\mathrm{DMSO}-d_{6}\right) \delta: 3.70\left(2 \mathrm{H}, \mathrm{s}, \mathrm{CH}_{2} \mathrm{CN}\right), 4.61(2 \mathrm{H}, \mathrm{s}$, $\left.\mathrm{CH}_{2} \mathrm{CO}\right), 7.87-7.96(4 \mathrm{H}, \mathrm{m}, \mathrm{ArH}), 11.66(1 \mathrm{H}$, brs, $1 \mathrm{NH}, \mathrm{ex}-$ changeable); IR (KBr) v: 3332, $3249(\mathrm{NH}), 3034\left(\mathrm{C}-\mathrm{H}_{\text {arom }}\right)$, 2973, $2932\left(\mathrm{C}-\mathrm{H}_{\text {aliph }}\right), 2260(\mathrm{CN}), 1777,1717(\mathrm{C}=\mathrm{O}), 1230$ $(\mathrm{C}=\mathrm{S}), 1467\left(\delta_{\mathrm{s}} \mathrm{CH}_{2}\right), 757 \delta_{4 \mathrm{H}}$; MS (70 eV) m/z (\%): $327\left(\mathrm{M}^{+}\right.$, 1), 305 (3), 278 (4), 263 (7), 238 (11), 223 (27), 204 (9), 177 (50), 160 (100), 124 (20), 111 (23), 97 (63), 83 (42); Anal. Calcd for $\mathrm{C}_{14} \mathrm{H}_{9} \mathrm{~N}_{5} \mathrm{O}_{3} \mathrm{~S}$ (327.32): C, 51.37; H, 2.77; N, 21.40. Found $\mathrm{C}$, 51.21; H, 2.65; N, 21.63\%.

1-(2-(1,3-Dioxoisoindolin-2-yl)acetyl)thiourea (7)

Seventy-eight percent yield; yellow crystals; mp $250-252^{\circ} \mathrm{C}$ $(\mathrm{EtOH}) ;{ }^{1} \mathrm{H}-\mathrm{NMR}\left(\mathrm{DMSO}-d_{6}\right) \delta: 4.66\left(2 \mathrm{H}, \mathrm{s}, \underline{\mathrm{CH}}_{2} \mathrm{CO}\right), 6.00$ (2H, brs, $\mathrm{NH}_{2}$, exchangeable), 7.86-7.92 (4H, $\left.\mathrm{m}, \mathrm{ArH}\right)$, for 7a: $13.64(1 \mathrm{H}$, brs, NH, exchangeable), for $7 \mathbf{b}: 9.89(1 \mathrm{H}$, brs, $\mathrm{SH}$, exchangeable); ${ }^{13} \mathrm{C}-\mathrm{NMR}$ (DMSO- $\left.d_{6}\right) \delta: 41.42\left(\mathrm{CH}_{2}\right)$, ar-C [123.42 (2CH), 131.47 (2C), $134.80(2 \mathrm{CH})], 167.24(\mathrm{C}=\mathrm{O})$, $168.78(2 \mathrm{C}=\mathrm{O}), 182.68(\mathrm{C}=\mathrm{S})$; IR $(\mathrm{KBr}) v: 3209,3177(\mathrm{NH})$, 3045, $3021\left(\mathrm{C}-\mathrm{H}_{\text {arom }}\right), 2963,2924\left(\mathrm{C}-\mathrm{H}_{\text {aliph }}\right), 1769,1736,1702$ $(\mathrm{C}=\mathrm{O}), 1197(\mathrm{CS}), 1453\left(\delta_{\mathrm{s}} \mathrm{CH}_{2}\right), 760 \delta_{4 \mathrm{H}} ; \mathrm{MS}(70 \mathrm{eV}) \mathrm{m} / \mathrm{z}(\%)$ : $263\left(\mathrm{M}^{+}\right.$, 4), 247 (8), 230 (12), 203 (5), 188 (65), 160 (100); Anal. Calcd for $\mathrm{C}_{11} \mathrm{H}_{9} \mathrm{~N}_{3} \mathrm{O}_{3} \mathrm{~S}$ (263.27): C, 50.18; H, 3.45; N, 15.96. Found C, 49.87; H, 3.32; N, 15.65\%.

2-(1,3-Dioxoisoindolin-2-yl)- $N$-(5-thioxo-4,5-dihydro1H-1,2,4-triazol-3-yl)acetamide (9)

Eighty-three percent yield; yellow crystals; mp $>300^{\circ} \mathrm{C}$ $\left(N, N\right.$-dimethylformamide (DMF)); ${ }^{1} \mathrm{H}-\mathrm{NMR}\left(\mathrm{DMSO}-d_{6}\right) \quad \delta$ : 4.60 (2H, s, $\left.\underline{\mathrm{CH}}_{2} \mathrm{CO}\right), 7.86-7.94$ (4H, m, ArH), 10.69, 11.90, 13.02 (3H, 3brs, 3NH, exchangeable); ${ }^{13} \mathrm{C}-\mathrm{NMR}$ (DMSO- $d_{6}$ ) $\delta$ : $40.4\left(\mathrm{CH}_{2}\right)$, ar-C [123.10 (2CH), $\left.131.57(2 \mathrm{C}), 134.78(2 \mathrm{CH})\right]$, $154.55(\mathrm{C}=\mathrm{N}), 165.61(\mathrm{C}=\mathrm{O}), 167.16(2 \mathrm{C}=\mathrm{O}), 167.27(\mathrm{C}=\mathrm{S}) ; v$ : 3260, 3222, $3173(\mathrm{NH}), 3018\left(\mathrm{C}-\mathrm{H}_{\text {arom }}\right), 2918\left(\mathrm{C}-\mathrm{H}_{\text {aliph }}\right), 1774$, $1710(\mathrm{C}=\mathrm{O}), 1189(\mathrm{C}=\mathrm{S}), 1473\left(\delta_{\mathrm{s}} \mathrm{CH}_{2}\right), 748 \delta_{4 \mathrm{H}} ; \mathrm{MS}(70 \mathrm{eV})$ m/z (\%): $303\left(\mathrm{M}^{+*}, 0.5\right), 270$ (0.5), 188 (0.5), 160 (1), 149
(8),129 (8), 115 (3), 109 (8), 81 (44), 69 (100), 55 (47); Anal. Calcd for $\mathrm{C}_{12} \mathrm{H}_{9} \mathrm{~N}_{5} \mathrm{O}_{3} \mathrm{~S}$ (303.30): C, 47.52; H, 2.99; N, 23.09. Found C, 47.23; H, 2.81; N, 22.77\%.

Ethyl 2-((2-(1,3-Dioxoisoindolin-2-yl)acetyl)carbamothioyl)hydrazine Carboxylate (10)

Seventy-eight percent yield; colorless crystals; mp $175-177^{\circ} \mathrm{C}$ (benzene-ethanol mixture); ${ }^{1} \mathrm{H}-\mathrm{NMR}$ (DMSO$\left.d_{6}\right) \delta: 1.18\left(3 \mathrm{H}, \mathrm{t}, J=7.2,6.9 \mathrm{~Hz}, \underline{\mathrm{CH}}_{3} \mathrm{CH}_{2} \mathrm{O}-\right), 4.06(2 \mathrm{H}, \mathrm{q}$, $\left.J=7.2 \mathrm{~Hz}, \mathrm{CH}_{3} \underline{\mathrm{CH}}_{2} \mathrm{O}-\right), 4.54\left(2 \mathrm{H}, \mathrm{s}, \underline{\mathrm{CH}}_{2} \mathrm{CO}\right), 7.87-7.95(4 \mathrm{H}$, $\mathrm{m}, \mathrm{ArH}), 9.69$, (1H, brs, NHCS, exchangeable), $11.27(1 \mathrm{H}$, brs, NㅂCOOEt, exchangeable), $11.89(1 \mathrm{H}$, brs, CON $\underline{H C S}$, exchangeable); IR (KBr) v: 3266, $3140(\mathrm{NH}), 3034\left(\mathrm{C}-\mathrm{H}_{\text {arom }}\right)$, 2982, $2932\left(\mathrm{C}-\mathrm{H}_{\text {aliph }}\right), 1777,1711(\mathrm{C}=\mathrm{O}), 1188(\mathrm{C}=\mathrm{S}), 1470$ $\left(\delta_{\mathrm{s}} \mathrm{CH}_{2}\right), 750 \delta_{4 \mathrm{H}} ; \mathrm{MS}(70 \mathrm{eV}) \mathrm{m} / \mathrm{z}(\%): 350\left(\mathrm{M}^{+}, 20\right), 332$ (2), 304 (3), 288 (5), 262 (24), 228 (31), 188 (21), 177 (18), 160 (100), 133 (33), 104 (75), 77 (34); Anal. Calcd for $\mathrm{C}_{14} \mathrm{H}_{14} \mathrm{~N}_{4} \mathrm{O}_{5} \mathrm{~S}$ (350.35): C, 47.99; H, 4.03; N, 15.99. Found C, 47.67; H, 3.88; N, $15.82 \%$.

6-((1,3-Dioxoisoindolin-2-yl)methyl)-4-oxo-2-thioxo-1,2,3,4tetrahydropyrimidine-5-carboxamide (13)

Sixty-eight percent yield; yellow crystals; mp $280-282^{\circ} \mathrm{C}$ $(\mathrm{EtOH}) ;{ }^{1} \mathrm{H}-\mathrm{NMR}$ (DMSO- $\left.d_{6}\right) \delta: 4.15\left(2 \mathrm{H}, \mathrm{s}, \underline{\mathrm{CH}}_{2} \mathrm{CO}\right), 7.81$, 7.63 (2H, two brs, $\mathrm{NH}_{2}$, exchangeable), 7.84-7.92 $(4 \mathrm{H}, \mathrm{m}$, ArH), 9.87 (1H, brs, NHCS exchangeable), $13.63(1 \mathrm{H}$, brs, CSNHCO exchangeable); ${ }^{13} \mathrm{C}-\mathrm{NMR}$ (DMSO- $\left.d_{6}\right) \quad \delta: 40.31$ $\left(\mathrm{CH}_{2}\right)$, ar-C [123.11 (2CH), $\left.131.70(2 \mathrm{C}), 134.47(2 \mathrm{CH})\right], 167.51$ $(2 \mathrm{C}=\mathrm{C}), 167.86,(3 \mathrm{CO}), 171.80\left(\mathrm{CO}_{\text {amide }} \& \mathrm{C}=\mathrm{S}\right)$; IR $(\mathrm{KBr})$ v: 3468, 3350, 3142, $3102(\mathrm{NH}), 3051\left(\mathrm{C}-\mathrm{H}_{\text {arom }}\right), 2923,2881$ $\left(\mathrm{C}-\mathrm{H}_{\text {aliph }}\right), 1765,1715,1694(\mathrm{C}=\mathrm{O}), 1655(\mathrm{C}=\mathrm{C}), 1138(\mathrm{C}=\mathrm{S})$, $1466\left(\delta_{\mathrm{s}} \mathrm{CH}_{2}\right), 734 \delta_{4 \mathrm{H}}$; MS (70 eV) $m / z(\%): 330\left(\mathrm{M}^{+}, 2\right), 318$ (3), 280 (1), 275 (2), 256 (1), 193 (22), 185 (20), 157 (25), 146 (18), 137 (31), 136 (27), 123 (18),73 (51), 69 (100); Anal. Calcd for $\mathrm{C}_{14} \mathrm{H}_{10} \mathrm{~N}_{4} \mathrm{O}_{4} \mathrm{~S}$ (330.32): C, 50.91; H, 3.05; N, 16.96. Found C, 50.78; H, 2.79; N, 16.84\%.

Reaction of Compounds $\mathbf{3}$ and $\mathbf{1 0}$ with Ethanolic Hydrochloric Acid Mixture

Syntheses of 1,2,4-Triazole Derivatives 4 and $\mathbf{1 2}$

General Procedure A solution of compound $\mathbf{3}$ and/or $\mathbf{1 0}$ $(0.5 \mathrm{~g})$ in ethanol $(30 \mathrm{~mL})$, and $3 \mathrm{M}$ hydrochloric acid $(5 \mathrm{~mL})$ was refluxed for $3 \mathrm{~h}$. A solid product was obtained during reflux in case of reaction of $\mathbf{3}$, while for the reaction of $\mathbf{1 0}$ the solvent was removed by vacuum-distillation. The solid products were filtered off and recrystallized from suitable solvents to give compounds $\mathbf{4}$ and $\mathbf{1 2}$.

Ethyl 2-(4-(2-(1,3-Dioxoisoindolin-2-yl)acetyl)-5-mercapto4H-1,2,4-triazol-3-yl)acetate (4)

Eighty-one percent yield; yellow crystals (benzene); mp $84-86^{\circ} \mathrm{C}$; ${ }^{1} \mathrm{H}-\mathrm{NMR}$ (DMSO- $\left.d_{6}\right) \quad \delta: 1.20(3 \mathrm{H}, \mathrm{t}, J=6.9 \mathrm{~Hz}$, $\left.\mathrm{CH}_{3} \mathrm{CH}_{2} \mathrm{O}\right), 3.64\left(2 \mathrm{H}, \mathrm{s}, \mathrm{CH}_{2} \mathrm{COOEt}\right), 4.16(2 \mathrm{H}, \mathrm{q}, J=6.9 \mathrm{~Hz}$, $\left.\mathrm{CH}_{3} \mathrm{CH}_{2} \mathrm{O}\right), 4.42\left(2 \mathrm{H}, \mathrm{s}, \mathrm{CH}_{2} \mathrm{CO}\right), 7.16(1 \mathrm{H}$, br s, SH, exchangeable), 7.87-7.96 (4H, m, ArH); ${ }^{13} \mathrm{C}-\mathrm{NMR}$ (DMSO- $\left.d_{6}\right) \delta: 13.94$ $\left(\underline{\mathrm{CH}}_{3} \mathrm{CH}_{2} \mathrm{O}\right), 40.31\left(2 \mathrm{CH}_{2} \mathrm{CO}\right), 61.36\left(\mathrm{CH}_{3} \underline{\mathrm{CH}}_{2} \mathrm{O}\right)$, ar- $\mathrm{C}[123.42$ $(2 \mathrm{CH}), 131.27(2 \mathrm{C}), 134.86(2 \mathrm{CH})], 144.69(2 \mathrm{C}=\mathrm{N}), 167.05$, 167.48, $176.33(4 \mathrm{C}=\mathrm{O})$; IR $(\mathrm{KBr}) v$ : $3229(\mathrm{NH}), 3099,3046$ $\left(\mathrm{C}-\mathrm{H}_{\text {arom }}\right), 2988,2964,2944\left(\mathrm{C}-\mathrm{H}_{\text {aliph }}\right), 1775,1722,1690$ $(\mathrm{C}=\mathrm{O}), 1611(\mathrm{C}=\mathrm{N}), 1470,1418\left(\delta_{\mathrm{s}} \mathrm{CH}_{2}\right), 743 \delta_{4 \mathrm{H}} ; \mathrm{MS}(70 \mathrm{eV})$ m/z (\%): $374\left(\mathrm{M}^{+}, 3\right)$; 301 (9), 288 (6), 260 (12), 214 (84), 203 (5), 188 (18), 174 (23), 160 (100), 142 (92), 104 (63), 77 (74); Anal. Calcd for $\mathrm{C}_{16} \mathrm{H}_{14} \mathrm{~N}_{4} \mathrm{O}_{5} \mathrm{~S}$ (374.37): C, 51.33; H, 3.77; N, 14.97. Found C, 51.18; H, 3.56; N, 14.74\%. 
2-(2-(3,5-Diethoxy-4H-1,2,4-triazol-4-yl)-2-oxoethyl)isoindoline-1,3-dione (12)

Sixty-eight percent yield; colorless crystals (ethanol); mp $110-112^{\circ} \mathrm{C} ;{ }^{1} \mathrm{H}-\mathrm{NMR}\left(\mathrm{DMSO}-d_{6}\right) \delta: 1.20(6 \mathrm{H}, \mathrm{t}, J=7.2,6.9 \mathrm{~Hz}$, $\left.2 \mathrm{CH}_{3} \mathrm{CH}_{2}\right), 4.15\left(4 \mathrm{H}, \mathrm{q}, J=7.2 \mathrm{~Hz}, 2 \mathrm{CH}_{3} \mathrm{CH}_{2}\right), 4.41(2 \mathrm{H}, \mathrm{s}$, $\left.\mathrm{CH}_{2} \mathrm{CO}\right), 7.87-7.96(4 \mathrm{H}, \mathrm{m}, \mathrm{ArH}) ;{ }^{13} \mathrm{C}-\mathrm{NMR}\left(\mathrm{DMSO}-d_{6}\right) \delta$ : $13.91\left(2 \mathrm{CH}_{3} \mathrm{CH}_{2} \mathrm{O}\right), 40.35\left(\mathrm{CH}_{2} \mathrm{CO}\right), 61.36\left(2 \mathrm{CH}_{3} \mathrm{CH}_{2} \mathrm{O}\right)$, ar- $\mathrm{C}$ [123.42 (2CH), $131.29(2 \mathrm{C}), 134.85(2 \mathrm{CH})], 159.73(2 \mathrm{C}=\mathrm{N})$, $167.05(\mathrm{C}=\mathrm{O}), 167.47(2 \mathrm{C}=\mathrm{O})$; IR $(\mathrm{KBr}) v$ : 3099, $3046(\mathrm{C}-$ $\left.\mathrm{H}_{\text {arom }}\right), 2945,2906\left(\mathrm{C}-\mathrm{H}_{\text {aliph }}\right), 1775,1726,1691(\mathrm{C}=\mathrm{O}), 1610$ $(\mathrm{C}=\mathrm{N}), 1470,1417\left(\delta_{\mathrm{s}} \mathrm{CH}_{2}\right), 743 \delta_{4 \mathrm{H}} ; \mathrm{MS}(70 \mathrm{eV}) \mathrm{m} / \mathrm{z}(\%): 344$ $\left(\mathrm{M}^{+}, 0\right), 296$ (3), 282 (4), 264 (9), 234 (49), 219 (7), 188 (17), 174 (16), 161 (64), 160 (100), 133 (33), 104 (31), 76 (24); Anal. Calcd for $\mathrm{C}_{16} \mathrm{H}_{16} \mathrm{~N}_{4} \mathrm{O}_{5}$ (344.32): C, 55.81; H, 4.68; N, 16.27. Found C, 55.59; H, 4.65; N, 15.91\%.

\section{Reaction of Compound 3 with Ethanolic $\mathbf{N a O H}$}

Synthesis of $(E / Z)-2-(4-(2-(1,3-D i o x o i s o i n d o l i n-2-y l) a c e t y l)-$ 5-thioxo-1,2,4-triazolidin-3-ylidene)acetic Acid (6)

To a solution of compound $3(1 \mathrm{~g})$ in ethanol $(30 \mathrm{~mL}), 3 \mathrm{M}$ sodium hydroxide $(5 \mathrm{~mL})$ was added. The reaction mixture was refluxed for $1 \mathrm{~h}$, then vacuum-distilled to $c a$. half volume, cooled to ambient temperature and acidified with dilute hydrochloric acid. The precipitated solid was collected, and recrystallized from ethanol to give compound 6: $83 \%$ yield; pale yellow crystals; mp $285-286^{\circ} \mathrm{C} ;{ }^{1} \mathrm{H}-\mathrm{NMR}\left(\mathrm{DMSO}-d_{6}\right) \delta$ : $4.38\left(2 \mathrm{H}, \mathrm{s}, \mathrm{CH}_{2} \mathrm{CO}\right)$, for $Z$-isomer $(97 \%) 5.94(1 \mathrm{H}, \mathrm{s}, \mathrm{CH}=)$, for $E$-isomer $(3 \%) 5.88(1 \mathrm{H}, \mathrm{s}, \mathrm{CH}=), 7.52-7.97(4 \mathrm{H}, \mathrm{m}, \mathrm{ArH})$, $8.83(1 \mathrm{H}$, brs, NHNHCS, exchangeable), $11.76(1 \mathrm{H}$, brs, NHNHCS, exchangeable), $13.51(1 \mathrm{H}$, brs, $\mathrm{OH}$, exchangeable); ${ }^{13} \mathrm{C}-\mathrm{NMR}\left(\mathrm{DMSO}-d_{6}\right) \delta$ : $40.87\left(\mathrm{CH}_{2} \mathrm{CO}\right), 85.82(\mathrm{CH}=\mathrm{C})$, ar- $\mathrm{C}$ [123.44 (2CH), $131.21(2 \mathrm{C}), 134.74(2 \mathrm{CH})], 151.92(\mathrm{CH}=\underline{\mathrm{C}})$, $166.22(\mathrm{C}=\mathrm{S}), 167.12,167.28,167.77,168.81(4 \mathrm{C}=\mathrm{O})$; IR $(\mathrm{KBr})$ v: 3521-2488 (br OH), $3299(\mathrm{NH}), 1775,1722,1677(\mathrm{C}=\mathrm{O})$, $1625(\mathrm{C}=\mathrm{N}), 1255(\mathrm{C}=\mathrm{S}), 1441\left(\delta_{\mathrm{s}} \mathrm{CH}_{2}\right), 760 \delta_{4 \mathrm{H}} ; \mathrm{MS}(70 \mathrm{eV})$ $m / z$ (\%): $346\left(\mathrm{M}^{+\cdot}, 0.05\right), 327$ (7), 313 (2), 268 (10), 197 (30), 160 (60), 133 (14), 104 (100) 84 (32), 76 (72); Anal. Calcd for $\mathrm{C}_{14} \mathrm{H}_{10} \mathrm{~N}_{4} \mathrm{O}_{5} \mathrm{~S}$ (346.32): C, 48.55; H, 2.91; N, 16.18. Found $\mathrm{C}$, 48.67; H, 2.75; N, 15.83\%.

Reaction of Isothiocyanate (1) with the Carbon Nucleophiles

Syntheses of Pyrimidine $\mathbf{1 4}$ and 1,3-Oxazine 16

General Procedure A solution of isothiocyanate (1) ( $3 \mathrm{mmol})$ and 2-cyanoacetamide $(3 \mathrm{mmol})$ in a dry acetonitrile $(30 \mathrm{~mL})$ was refluxed for $3 \mathrm{~h}$ in the presence of a catalytic amount of piperidine $(3 \mathrm{mmol})$. The reaction mixture was cooled to ambient temperature. A solid product was obtained; filtered off and washed with dilute $\mathrm{HCl}$, then water several times to give compounds $\mathbf{1 4}$. The same procedure was done with $p$-nitrobenzyl cyanide to give compounds $\mathbf{1 6}$. The solid product obtained for each reaction was recrystallized from a suitable solvent to give the corresponding compound.

2-((5-(Amino(piperidin-1-yl)methylene)-6-oxo-2-thioxo1,2,5,6-tetrahydropyrimidin-4-yl)methyl)isoindoline-1,3-dione (14)

Sixty-seven percent yield; colerless crystals; mp $238-240^{\circ} \mathrm{C}$ (EtOH); ${ }^{1} \mathrm{H}-\mathrm{NMR}$ (DMSO- $\left.d_{6}\right) \delta: 1.46-1.65$ (6H, m, 3CH $\mathrm{CH}_{2}$ pip.), $3.2\left(4 \mathrm{H}, \mathrm{s}, \mathrm{CH}_{2} \mathrm{NCH}_{2}\right.$, pip.), $4.15\left(2 \mathrm{H}, \mathrm{s}, \mathrm{CH}_{2} \mathrm{CO}\right), 7.84-7.92$ $(4 \mathrm{H}, \mathrm{m}, \mathrm{ArH})$, for 14a: $3.28\left(2 \mathrm{H}, \mathrm{brs}, \mathrm{NH}_{2}\right.$, exchangeable), 10.34 (1H, brs, CONHCS, exchangeable), for $\mathbf{1 4 b}: 7.19(1 \mathrm{H}$, brs, $\mathrm{SH}$, exchangeable), 7.65 (1H, brs, $\mathrm{OH}$, exchangeable), 8.21 $(1 \mathrm{H}$, brs, $\mathrm{NH}=$, exchangeable); IR (KBr) v: 3421, 3318, 3276,
3208, $3119(\mathrm{NH}), 3039\left(\mathrm{C}-\mathrm{H}_{\text {arom }}\right), 2936,2856,2771,2711(\mathrm{C}-$ $\left.\mathrm{H}_{\text {aliph }}\right), 1770,1721,1681(\mathrm{C}=\mathrm{O}), 1615,1545(\mathrm{C}=\mathrm{N}), 1139(\mathrm{CS})$, $1420\left(\delta_{\mathrm{s}} \mathrm{CH}_{2}\right), 736 \delta_{4 \mathrm{H}} ; \mathrm{MS}(70 \mathrm{eV}) \mathrm{m} / z(\%): 397\left(\mathrm{M}^{+*}, 0\right)$, $380\left(\mathrm{M}^{+\cdot}-\mathrm{OH}, 1\right), 258$ (72), 256 (100), 192 (41), 160 (91), 128 (68), 96 (21), 64 (60); Anal. Calcd for $\mathrm{C}_{19} \mathrm{H}_{19} \mathrm{~N}_{5} \mathrm{O}_{3} \mathrm{~S}$ (397.45): C, 57.42; H, 4.82; N, 17.62. Found C, 57.14; H, 4.65; N, 17.29\%.

2-((6-Imino-5-(4-nitrophenyl)-4-thioxo-5,6-dihydro-4H-1,3oxazin-2-yl)methyl)isoindoline-1,3-dione (16)

Sixty-seven percent yield; yellow crystals; $295-296^{\circ} \mathrm{C}$ (ethanol); ${ }^{1} \mathrm{H}-\mathrm{NMR}\left(\mathrm{DMSO}-d_{6}\right) \delta: 4.16\left(2 \mathrm{H}, \mathrm{s}, \mathrm{CH}_{2}\right), 7.84-7.96$ $(8 \mathrm{H}, \mathrm{m}, \mathrm{ArH})$; for isomer 16b: $7.19\left(2 \mathrm{H}\right.$, brs, $\mathrm{NH}_{2}$, exchangeable); for isomer 16a: $4.61\left(1 \mathrm{H}, \mathrm{s}, \mathrm{H}_{\mathrm{m}}\right), 11.64(1 \mathrm{H}$, brs, $=\mathrm{NH}$, exchangeable); IR (KBr) v: 3425, 3324, $3207(\mathrm{NH}), 3098,3086$ $\left(\mathrm{C}-\mathrm{H}_{\text {arom }}\right), 1776,1725(\mathrm{C}=\mathrm{O}), 1609(\mathrm{C}=\mathrm{N}), 1195(\mathrm{CS}), 1467$ $\left(\delta_{\mathrm{s}} \mathrm{CH}_{2}\right) ; \mathrm{MS}(70 \mathrm{eV}) \mathrm{m} / \mathrm{z}(\%): 408\left(\mathrm{M}^{+\cdot}, 5\right), 389(6), 370$ (4), 358 (5), 313 (7), 236 (8), 222 ((6), 183 (7), 161 (6), 138 (14), 97 (69), 57 (100); Anal. Calcd for $\mathrm{C}_{19} \mathrm{H}_{12} \mathrm{~N}_{4} \mathrm{O}_{5} \mathrm{~S}$ (408.39): C, 55.88; H, 2.96; N, 13.72. Found C, 55.62; H, 2.78; N, 13.51\%.

Assessment of the Antimicrobial Activity Using the Agar Well Diffusion Technique The chemically synthesized compounds were screened for their antibacterial and antifungal activities using the agar well diffusion technique. ${ }^{28)}$ The microorganisms (reference and clinical isolates) used include $E$. coli (ATCC-25923), Salmonella typhi, Staphylococcus aureus (ATCC-25923), Candida albicans (ATCC-10231) and Aspergillus flavus. For the antibacterial assay, a standard inoculum $\left(10^{5}\right.$ colony forming unit $\left.(\mathrm{CFU}) / \mathrm{mL}\right)$ was distributed on the surface of the agar plates using a sterile glass spreader, whereas for the antifungal assay a loopful of a particular fungal isolate was transferred to $3 \mathrm{~mL}$ sterile saline to get a suspension of the corresponding species; $0.1 \mathrm{~mL}$ of the spore suspension was distributed on the surface of sterile Sabouraud dextrose agar plates. Six millimeter diameter wells were punched in the agar media and filled with $100 \mu \mathrm{L}$ of the tested chemical compound $(500 \mu \mathrm{g} / \mathrm{mL}$ in DMSO) which is previously sterilized through 0.45 sterile membrane filter. The plates were kept at room temperature for $1-2 \mathrm{~h}$ then incubated at $37^{\circ} \mathrm{C}$ for $24 \mathrm{~h}$ for bacteria and at $30^{\circ} \mathrm{C}$ for $4 \mathrm{~d}$ for fungi. Commercial antibiotic discs were used as positive reference standard to determine the sensitivity of the strains. ${ }^{29}$ )

Determination of the MIC of the Chemical Compounds Compounds inhibiting the growth of the above microorganisms were tested for their MIC by the broth dilution method. ${ }^{30}$ ) The nutrient broth and the yeast extract broth media containing $1 \mathrm{~mL}$ of the serial dilutions of the tested compounds $(3.125,6.25,12.5,25.50 \mu \mathrm{g} / \mathrm{mL})$ were inoculated with the microbial strains, the bacterial cultures were incubated at $37^{\circ} \mathrm{C}$ for $24 \mathrm{~h}$, whereas the fungal ones were incubated at $30^{\circ} \mathrm{C}$ for $48 \mathrm{~h}$. The lowest concentration required to arrest the microbial growth was regarded as the MIC of the tested compounds.

\section{Conclusion}

The electron-withdrawing power of the phtalimido moiety enhances the reactivity of both isothiocyanate function and carbonyl group of $\mathbf{1}$. It is observed from the mentioned reactions that the nucleophilic addition proceeds entirely at the isothiocyanate function. Simultaneous or subsequent cyclization of obtained adduct produced the target heterocycles. The synthesized heterocycles showed promising antimicrobial activity comparable to sulphamethoxazole and fluconazole. This may be attributed to their attachments to phthalimido moiety 
at their structures.

Acknowledgments The authors are indebted to Dr. Nashwa, A. Ahmed (Department of Microbiology, Faculty of Applied Medical Sciences, 6 October University, Giza, Egypt) and Prof. Yousseria, M. Shetaia (Department of Microbiology, Faculty of Science, Ain Shams University, Cairo, Egypt) for assessment of the antimicrobial activity of the synthesized compounds at their laboratories.

Conflict of Interest The authors declare no conflict of interest.

\section{References}

1) Bhambi D., Salvi V. K., Bapna A., Pemawat G., Talesara G. L., Indian J. Chem., 48B, 697-704 (2009).

2) Akgün H., Karamelekoğlu I., Berk B., Kurnaz I., Sarıbıyık G., Öktem S., Kocagöz T., Bioorg. Med. Chem., 20, 4149-4154 (2012).

3) Spallarossa A., Cesarini S., Ranise A., Bruno O., Schenone S. La Colla P., Collu G., Sanna G., Secci B., Loddo R., Eur. J. Med. Chem., 44, 1650-1663 (2009).

4) Liu W. M., Henry J. Y., Meyer B., Bartlett J. B., Dalgleish A. G., Galustian C., Br. J. Cancer, 101, 803-812 (2009).

5) Lopez-Girona A., Mendy D., Ito T., Miller K., Gandhi A. K., Kang J., Karasawa S., Carmel G., Jackson P., Abbasian M., Mahmoudi A., Cathers B., Rychak E., Gaidarova S., Chen R., Schafer P. H., Handa H., Daniel T. O., Evans J. F., Chopra R., Leukemia, 26, 2326-2335 (2012).

6) Tetsuhashi M., Ishikawa M., Hashimoto M., Hashimoto Y., Aoyama H., Bioorg. Med. Chem., 18, 5323-5338 (2010).

7) Lima L. M., de Brito F. C. F., de Souza S. D., Miranda A. L. P., Rodrigues C. R., Fraga C. A. M., Barreiro E. J., Bioorg. Med. Chem. Lett., 12, 1533-1535 (2002).

8) Motoshima K., Sugita K., Hashimoto Y., Ishikawa M., Bioorg. Med. Chem. Lett., 21, 3041-3045 (2011).

9) Motoshima K., Noguchi-Yachide T. N., Sugita K., Hashimoto Y., Ishikawa M., Bioorg. Med. Chem., 17, 5001-5014 (2009).

10) Kamiński K., Obniska J., Wiklik B., Atamanyuk D., Eur. J. Med. Chem., 46, 4634-4641 (2011).
11) Manley-King C. I., Bergh J. J., Petzer J. P., Bioorg. Med. Chem., 19, 4829-4840 (2011).

12) Guzior N., Bajda M., Rakoczy J., Brus B., Gobec S., Malawska B., Bioorg. Med. Chem., 23, 1629-1637 (2015).

13) Singhal N., Sharma P. K., Dudhe R., Kumar N., J. Chem. Pharm. Res., 3, 126-133 (2011).

14) Kuchkova K., Aricu A., Secara E., Barba A., Vlad P., Ungur N., Tuchilus C., Shova S., Zbancioc G., Mangalagiu I. I., Med. Chem. Res., 23, 1559-1568 (2014).

15) Palmer B. D., Sutherland H. S., Blaser A., Kmentova I., Franzblau S. G., Wan B., Wang Y., Ma Z., Denny W. A., Thompson A. M., Thompson M. A., J. Med. Chem., 58, 3036-3059 (2015).

16) Korkmaz N., Obaidi O. A., Senturk M., Astley D., Ekinci D., Supuran C. T. J. Enzy Inhibi., Med. Chem., 30, 75-80 (2015).

17) Hemdan M. M., Phosphorus Sulfur Silicon Relat. Elem., 185, 620 627 (2010).

18) Fahmy A. F., Ali N., Abdelhamid H., Shiba S., Hemdan M. M., Phosphorus Sulfur Silicon Relat. Elem., 185, 1536-1542 (2010).

19) Hemdan M. M., El-Sayed A. A., J. Heterocyclic Chem. Early view (online version) 2015.

20) Hemdan M. M., Fahmy A. F., El-Sayed A. A., J. Chem. Res., 34, 219-221 (2010).

21) Hemdan M. M., Fahmy A. F., Ali N. F., Hegazi E., Abd-Elhaleem A., Chin. J. Chem., 25, 388-391 (2007).

22) Hemdan M. M., Fahmy A. F. M., Aly N. F., Hegazi I. A., El-Sayed A. A., Phosphorus, Sulfur, and Silicon, 187, 181-189 (2012).

23) Hemdan M. M., Abd El-Mawgoude K. H., Chem. Pharm. Bull., 63, 450-456 (2015)

24) Hemdan M. M., Abd El-Mawgoude K. H., Chem. Pharm. Bull., 63, 812-818 (2015).

25) Vol. 2, ed. by Drobnica L., Kristian P., Augustin J. A., Patai S., John Wiley \& Sons, New York, 1977, pp. 1003-1221.

26) Sharma S., Sulfur Rep., 8, 327-454 (1989).

27) Mukerjee A. K., Ashare R., Chem. Rev., 91, 1-24 (1991).

28) Naz S., Jabeen S., Ilyas S., Farkhanda M. F., Aslam F., Ali A., Pak. J. Bot., 42, 455-462 (2010).

29) Kandile N. G., Zaky H. T., Mohamed M. I., Ismaeel H. M., Ahmed N. A., J. Enzyme Inhib. Med. Chem., 27, 599-608 (2012).

30) Valgas C., de Souza S. M., Smânia E. F. A., Smânia A. Jr., Braz. J. Microbiol., 38, 369-380 (2007). 